\title{
Study and Performance Comparison of Geocross and GPSR
}

\author{
Mandar R.Pandharpurkar \\ M.B.E.S. College of Engineering, \\ Ambajogai.
}

\author{
Bankat M.Patil \\ M.B.E.S. College of Engineering, \\ Ambajogai.
}

\begin{abstract}
In this paper, we compared Greedy Perimeter Stateless Routing protocol(GPSR), a position-based routing which forwards a packet to an immediate neighbor, a greedy mode with Geocross, an event-driven geographic routing which removes cross-links dynamically to avoid routing loops in urban vehicular Ad Hoc Network (VANET), where the Packet Delivery Ratio (PDR) is consistently higher in Geocross and packet loss ratio is lower in Geocross than the GPSR.
\end{abstract}

\section{Keywords}

Greedy Mode, VANET, Geocross, GPSR.

\section{INTRODUCTION}

The growing spread of vehicles and traffic and the use of network require an efficient communication mode among the vehicles, which forms a highly dynamic vehicular Ad-Hoc Network. The position-based routings are suitable for such a highly dynamic VANET such as Greedy perimeter stateless routing, transfers the packets to the nearest neighbors by using greedy forwarding[1].

However, problems in particular, cross links can arise when nodes are highly mobile in the network. The nodes normally forms a planar graph with no cross-edges, where the packets transfers through the nodes from source to destination. The perimeter mode, where the planar graph is formed, the packet collision will be avoided in the planar graph.

In VANET, in the urban scenario, the roads forms a planar graph, intersection between the nodes forms an edge. The nodes can transfer packets as soon as they can form intersection with neighboring node, As the nodes are highly mobile in the network, the dynamic loops are naturally formed, with the possibility of cross-link between the nodes[1] . The Geocross, an event driven protocol removes these cross-links dynamically without any notification. Some position based routing protocol, Global Positioning System(GPS)[2] becomes a solution, but to remove the crosslinks in the dynamic loops requires the dynamic-loopdetection. AODV maintains routes for as long as the route is active. This includes maintaining a multicast tree for the life of the multicast group. Because the network nodes are mobile, it is likely that many link breakages along a route will occur during the lifetime of that route On demand protocols such as Ad-hoc on demand distance vector(AODV)[3] or Dynamic Source Routing(DSR)[4] suffer from flooding overhead, also these approaches do not maintain the performance when the number of nodes increases.

Greedy algorithms apply some type of greedy path finding heuristic that does not guarantee that a packet ultimately reaches its destination. These include the geographic distance routing (GEDIR) algorithm of Lin and Stojmenovi'c [5], compass routing algorithm of Basagni et al. [6].As the nodes are highly mobile, the greedy mode finds the neighboring node which in closest and transfers the packet, due to the highly mobility of node, the neighboring node may change. So the information of packet transfer to the corresponding node must be stored.

As the packets can be transferred to the neighboring nodes based on the position of the nodes present in the network, but the nodes are highly movable. The path from the source node to the destination node may change in the same network. So the packet needs to change the route when the node moves in the network, this generates cross links between the nodes while moving in the network.

The paper demonstrates a study on GPSR, a position-based routing which forwards a packet to an immediate neighbor, a greedy mode with Geocross, event-driven geographic routing which removes cross-links dynamically to avoid routing loops in urban VANET which uses greedy mode and perimeter forwarding mode and avoids the crosslink generated in the dynamic loop where the PDR is consistently higher in Geocross and packet loss ratio is lower in Geocross than the GPSR.

\section{GREEDY PERIMETER STATELESS ROUTING}

The GPSR is a position-based routing. The nodes forward the packets to an immediate neighbor which is geographically closer to the destination node. This mode is the Greedy Mode. Due to the highly mobility of nodes, a local maximum can occur in the Greedy mode. It requires some recovery algorithms like GOAFR[6].GPSR recovers using perimeter mode using right hand rule. Consider Fig.1, $\mathrm{S}$ is the source node which transfers the packet to the destination node $\mathrm{D}$. According to the right hand rule, source $S$ selects the node closest to it i.e. node X., through node $\mathrm{Y}$ the packet will reach at the destination $\mathrm{D}$.

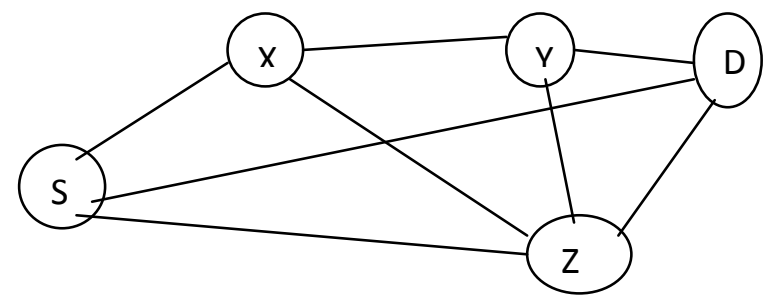

Fig 1: Demonstrating Perimeter forwarding mode using right hand rule. 


\section{GEOCROSS ALGORITHM}

Geo-cross is a geographic routing protocol, Greedy forwarding and perimeter forwarding modes are included.

The Geo-cross includes:

1. Cross-link detection and removal is done.

2. Cross-link removal is done only when it is necessary.

3. No state information should be kept in nodes.

The Geocross algorithm uses perimeter forwarding algorithm to transfer the packets to the neighboring node and the nodes are highly mobile which may generates cross-link in the dynamic loop, the check loop process algorithm is used. The cross-links are detected and removed with no message. The node information is not maintained in the nodes. The dynamic loops are detected and the path of the packet is stored in probe field. The Probe field stores the information of the nodes where the packet can travel, with this information route can be discovered. UR, the unroutable routes are stored and VF, visited faces where the packet transferred though which nodes is stored. VF stores the information about the nodes where the packet is transmitted.

The Packet format is

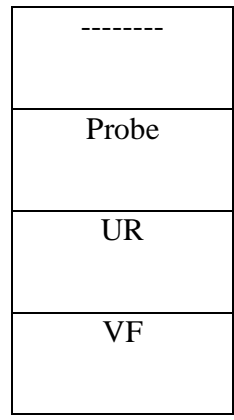

Fig 2: Packet Format[1]

In the greedy forwarding mode, Geocross forwards the packet to the junction closer to the destination. If local maximum occurred, recovery mode is used, i.e. perimeter forwarding is used. When the node receives a packet, it first check whether the loop with the help of probe field and checks if there is any cross link available. The node forwards the packet according to the loop indicated by the packet so that the detected crosslink can be removed in UR field. The Greedy forwarding mode is used which forwards the packet greedily towards the destination. If local maximum occurs, then recovery mode is used i.e. perimeter forwarding.

In perimeter forwarding, packet is sent to the node which is geographically closest to the current node. The dynamic loop detection with the highly mobile nodes where the routes, junctions and missing junctions are stored in probe field.

\subsection{Algorithm Perimeter Forward}

The perimeter forwarding algorithm transfers the packet to the nearest neighbor which is geographically closest to the current node and transfers the packet.

Input: current Hop, destination, Probe, UR, VF, node $\mathrm{x}$ that first enters into the perimeter mode
Output: next Hop

1 if

DIST(current Hop, destination) < DIST( $x$, destination)

2 then switch to Greedy Forwarding Mode

3 return current Hop

4 if current Hop not at junction

5 then nmax let the furthest potential forwarder along the current road in the same packet forwarding direction

6 if nmax exists

7 then return nmax

$8 \quad$ else mmax $\leftarrow$ the furthest Potential Forwarder along the current road in the opposite direction to the current packet forwarding direction

9 return mmax

10 else $\mathrm{PF} \leftarrow$ ordered list of potential forwarders by the right

Hand rule

11 forced_nextHop $\leftarrow$ call

CheckProcessLoop(Probe, UR)

12 if forced_nextHop exists

13 then $\mathrm{PF} \leftarrow$ (forcednextHop, $\mathrm{PF})$

14 for nextHop $\in \mathrm{PF}$

15 do road Link let road of current Hop to road of the next hop

16 next road let the road next Hop is on

17 if (road Link $\notin U R)$ and (next Road $\notin V F)$

18 then return next Hop

19 if current Hop is source node

$20 \quad$ then $\mathrm{VF} \leftarrow \mathrm{VF}\{$ next Road $\}$

21 return NULL [1]

\subsection{Algorithm Check Process Loop}

The check process loop algorithm checks the dynamic loops in the VANET and removes the crosslink if available.

Input: Probe, UR

Output: nextHop

1 loop $\leftarrow$ a routing loop enclosed by two junction IDs or

Roads in probe

2 rclink $\leftarrow$ set of cross-links in Probe

3 aclink $\leftarrow$ set of adjacent cross-links in Probe

4 if loop exists 


\section{RESULT DISCUSSION}

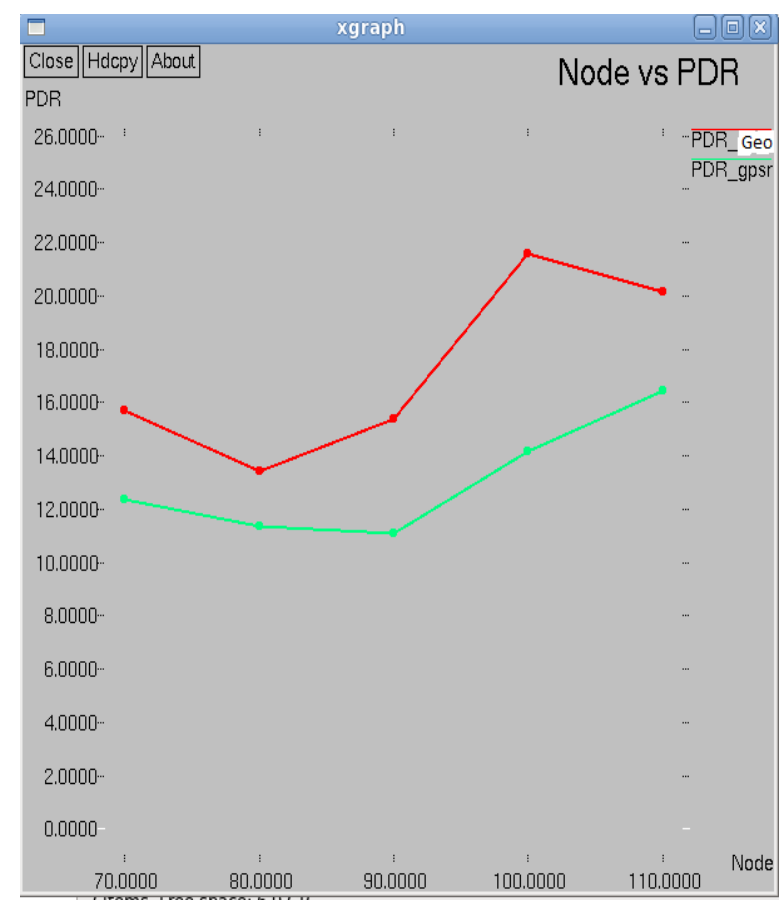

Fig 3. Number of Nodes V/s PDR

Table 1. Nodes V/S PDR

\begin{tabular}{|c|c|c|}
\hline Nodes & $\begin{array}{c}\text { PDR for } \\
\text { Geocross }\end{array}$ & PDR for GPSR \\
\hline 70 & 16.00 & 12.40 \\
\hline 80 & 13.80 & 11.90 \\
\hline 90 & 15.75 & 11.80 \\
\hline 100 & 22.00 & 14.10 \\
\hline 110 & 20.70 & 16.20 \\
\hline
\end{tabular}

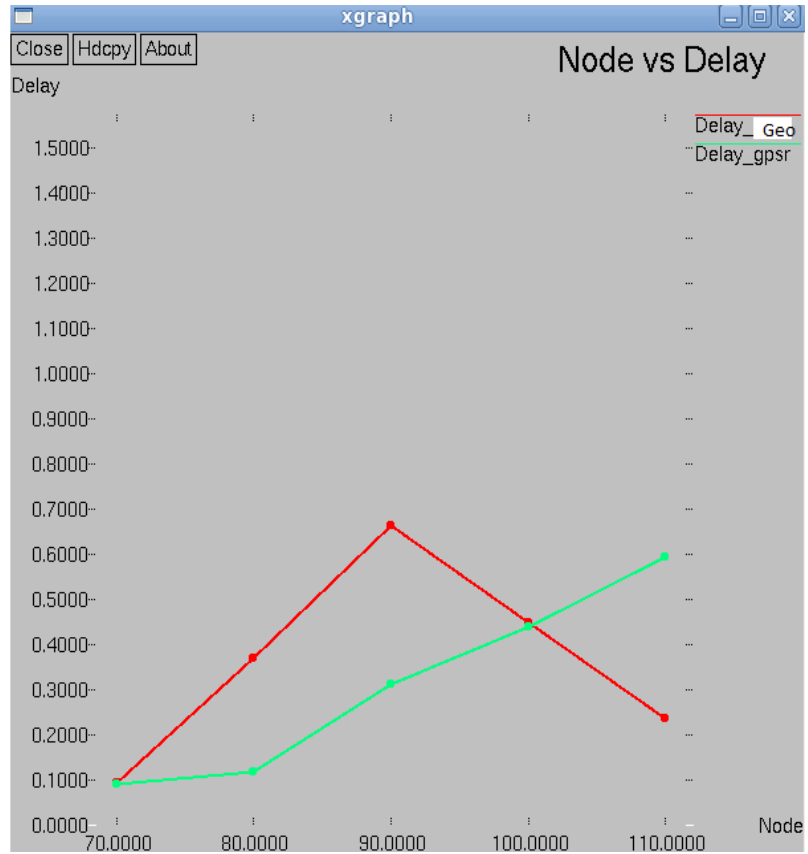

Fig 4. Nodes V/s Delay

Table 2. Nodes V/S Delay

\begin{tabular}{|c|c|c|}
\hline Nodes & $\begin{array}{c}\text { Delay for } \\
\text { Geocross }\end{array}$ & Delay for GPSR \\
\hline 70 & 0.1 & 0.1 \\
\hline 80 & 0.38 & 0.11 \\
\hline 90 & 0.69 & 0.31 \\
\hline 100 & 0.45 & 0.44 \\
\hline 110 & 0.21 & 0.59 \\
\hline
\end{tabular}

Fig 3 shows the PDR, Packet delivery ratio for the GPSR and Geo-cross, the scenario for 70 nodes to 110 nodes. PDR for the GPSR is comparably lower as compared to the Geo-cross, as the number of nodes increases, the PDR for Geocross is consistently higher than that of GPSR. The Table 1 shows the PDR for nodes 70 is 16.00 and for 110 nodes is 20.70 and for GPSR, for 70 nodes, it is 12.40 and for 110 , it is 16.20 .

Fig 4 shows the Delay for GPSR and Geo-cross in micro second, the scenario for 70 nodes to 110 nodes. Delay for the GPSR is comparably higher as compared to the Geo-cross, as the number of nodes increases, the delay for Geocross is consistently lower than that of GPSR. The Table 2 shows the delay for nodes 70 is 0.1 and for 110 nodes is 0.21 for Geocross and for GPSR, delay for nodes 70 is 0.1 and for 110 nodes delay is 0.59 .

The Table 1 shows the values for the PDR rate for Geocross and GPSR and Table 2 shows the values for delay. Number of nodes and the corresponding values for the PDR and delay are shown. 


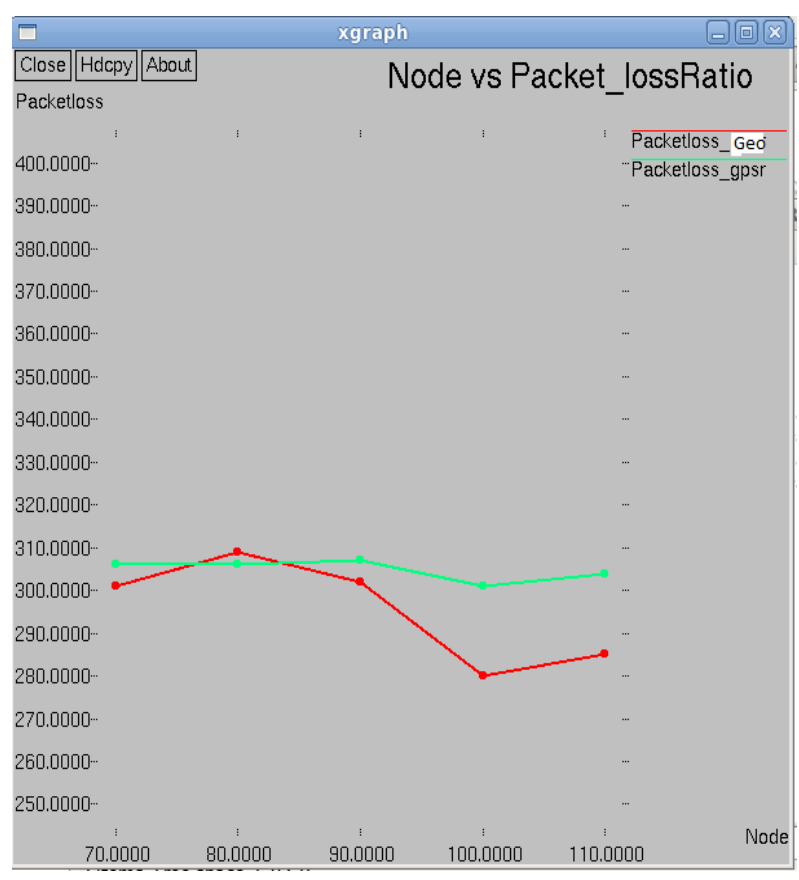

Fig 5. Node Vs Packet loss ratio

Table 3. Nodes V/S Packetloss Ratio

\begin{tabular}{|c|c|c|}
\hline Nodes & $\begin{array}{c}\text { Packet loss for } \\
\text { Geocross }\end{array}$ & $\begin{array}{c}\text { Packet loss for } \\
\text { GPSR }\end{array}$ \\
\hline 70 & 302.00 & 308.00 \\
\hline 80 & 309.00 & 307.00 \\
\hline 90 & 300.00 & 307.00 \\
\hline 100 & 276.00 & 304.00 \\
\hline 110 & 282.00 & 305.00 \\
\hline
\end{tabular}

Fig 5 shows the Packet loss ratio, Packet loss ratio for the GPSR and Geo-cross, the scenario for 70 nodes to 110 nodes. Packet loss for the GPSR is comparably higher as compared to the Geo-cross. The Table 3 shows the packet loss ratio for nodes 70 is 308.00 and for 110 nodes is 305.00 for GPSR and for Geocross, for 70 nodes, it is 302.00 and for 110 , it is 282.00

\section{CONCLUSION}

In this paper, the comparison between Geo-cross with 70 to 110 nodes, an event driven geographic routing protocol with the GPSR, a position-based protocol is shown. The packet Delivery Ratio, PDR for Geo-cross is consistently higher than that of GPSR, Greedy Perimeter Stateless Routing. Also the delay gets reduced in Geo-cross. The algorithm can be further improved to enhance the packet delivery ratio and packet loss can be reduced further.

\section{REFERENCES}

[1] Kevin C. Lee , Pei-Chun Cheng, Mario Gerla, "GeoCross: A geographic routing protocol in the presence of loops in urban scenarios", Ad Hoc Networks 8 (2010) 474-488

[2] R. Flury, R. Wattenhofer, MLS: an efficient location service for mobile ad hoc networks, in: MobiHoc '06: Proceedings of the 7th ACM International Symposium on Mobile Ad Hoc Networking and Computing, New York, NY, USA, 2006, pp. 226-237.

[3] C.E. Perkins, E.M. Royer, Ad hoc on-demand distance vector routing, in: Proceedings of the 2nd IEEE Workshop on Mobile Computing Systems and Applications, February 2005, pp. 90-100.

[4] D.B. Johnson, D.A. Maltz, Dynamic source routing in ad hoc wireless networks, in: Imielinski, Korth (Eds.), Mobile Computing, vol. 353, Kluwer Academic Publishers, 1996

[5] Y.T. Yu, M.F. Lau, "A comparison of MC/DC, MUMCUT and several other coverage criteria for logical decisions", Journal of Systems and Software, 2005, in press.

[6] Forman, G. 2003. An extensive empirical study of feature selection metrics for text classification. J. Mach. Learn. Res. 3 (Mar. 2003), 1289-1305.

[7] F. Kuhn, R. Wattenhofer, Y. Zhang, A. Zollinger, Geometric ad-hoc routing: of theory and practice, in: PODC '03: Proceedings of the Twenty-second Annual Symposium on Principles of Distributed Computing, New York, NY, USA, 2003, pp. 63-72.

[8] dash. <http://www.dash.net/>.

[9] Tiger, tiger/line and tiger-related products. U.S. Census Bureau. <http://www.census.gov/geo/www/tiger/>.

[10] P. Bose, P. Morin, I. Stojmenovic, J. Urrutia, Routing with guaranteeddelivery in ad hoc wireless networks, Wireless Networks 7 (6) (2001)609-616.

[11] P. Bose, P. Morin, I. Stojmenovic' , J. Urrutia, Routing with guaranteed delivery in ad hoc wireless networks, Wireless Networks 7 (6) (2001)609-616.

[12] Brown, L. D., Hua, H., and Gao, C. 2003. A widget framework for augmented interaction in SCAPE.

[13] S. Basagni, I. Chlamtac, V.R. Syrotiuk and B.A. Woodward, A distance routing effect algorithm for mobility (DREAM), in: ACM/IEEE International Conference on Mobile Computing and Networking (Mobicom'98) (1998) pp. 76-84.

[14] Lin and I. Stojmenovi'c, Geographic distance routing in ad hoc wireless networks, Technical report TR-98-10, SITE, University of Ottawa (December 1998). 